\title{
A Sensitivity Dosimetry Study of the Setup Uncertainties during Machine Commissioning and Annual QA
}

\author{
Vi Nhan Nguyen1', Brian Wang², Chengyu Shi \\ ${ }^{1}$ INPhysics, Zionsville, IN, USA \\ ${ }^{2}$ James Brown Cancer Center, The University of Louisville, Louisville, KY, USA \\ ${ }^{3}$ Memorial Sloan Kettering Cancer Center, Basking Ridge, NJ, USA \\ Email: vi.nhan.nguyen1@gmail.com, brian.wang@louisville.edu, shic@mskcc.org
}

How to cite this paper: Nguyen, V.N., Wang, B. and Shi, C.Y. (2016) A Sensitivity Dosimetry Study of the Setup Uncertainties during Machine Commissioning and Annual QA. International Journal of Medical Physics, Clinical Engineering and Radiation Oncology, 5, 329-347.

http://dx.doi.org/10.4236/ijmpcero.2016.54032

Received: October 13, 2016

Accepted: November 18, 2016

Published: November 21, 2016

Copyright $\odot 2016$ by authors and Scientific Research Publishing Inc. This work is licensed under the Creative Commons Attribution International License (CC BY 4.0).

http://creativecommons.org/licenses/by/4.0/

\section{Abstract}

This study is to investigate three common potential setup uncertainties during Linac commissioning and annual QA and to evaluate how these uncertainties propagate into the quality of beam profiles and patient dosimetry using gamma analysis. Three uncertainty scenarios were purposely introduced for gantry position tilted from $0^{\circ}-3^{\circ}$ (scenario 1 ), isocenter position misaligned from $0-6 \mathrm{~mm}$ (scenario 2 ) and SAD changed from $99.5-103 \mathrm{~cm}$ (scenario 3). A $60 \times 60 \times 60 \mathrm{~cm}^{3}$ water phantom cube was created to replicate a 3D water tank in VarianEclipse (V.11) treatment planning system (Varian Medical Systems, Palo Alto, CA). For each scenario, beam data profiles (crossline and diagonal) and PDD curves were calculated at different field sizes and depths for three energies: $6 \mathrm{MV}, 6 \mathrm{MV}$-FFF and $10 \mathrm{MV}$-FFF. Gamma analysis method was used to compare a total of 263 profiles to baseline using a $1 \% / 1 \mathrm{~mm}$ parameter with $90 \%$ gamma passing rate criteria. For scenario 1 , a $\geq 90 \%$ gamma passing rate and $\leq 1 \%$ dose difference were seen on both crossline and diagonal profiles, and PDD curves for gantry tilted up to $2^{\circ}$. For $3^{\circ}$ degree tilt, the gamma passing rate decreased to $\leq 90 \%$ at depth of $\geq 20 \mathrm{~cm}$ for $6 \mathrm{MV} / 6 \mathrm{MV}$-FFF and depth of $\geq 12 \mathrm{~cm}$ for $10 \mathrm{MV}$-FFF. For scenario 2 , a $\leq 90 \%$ gamma passing rate and $\geq 1 \%$ dose difference were seen at depths from $d_{\max }$ to $20 \mathrm{~cm}$ for all energies. For depths $\geq 20 \mathrm{~cm}$, mostly $\geq 90 \%$ gamma passing rate and $\leq 1 \%$ dose difference were seen. For scenario $3, a \geq 90 \%$ gamma passing rate and $\leq 1 \%$ dose difference were seen on $\leq 4 \mathrm{~mm}$ isocenter misalignments for all energies. In summary, gamma analysis of the beam profiles is a very sensitive test for SAD deviation scenarios and can reveal issues of sub millimeter setup uncertainty. However, it is not as sensitive for isocenter misalignment scenarios. The test is also more sensitive for FFF beams than flattening filter beams. 


\section{Keywords}

Linac Commissioning, Annual QA, Gamma Analysis, TG 142, Flattening Filter and Flattening Filter Free Beams

\section{Introduction}

The dosimetry accuracy is an important factor in the field of radiation therapy. The dosimetric accuracy is affected not only by the quality of the Linear Accelerator (Linac)'s commissioned radiation beam, but also on how well the commissioned Linac radiation beam can be maintained to achieve the same or similar radiation characteristics as commissioning time. A credential study conducted by the Imaging and Radiation Oncology Core (IROC) of MD Anderson (Houston, Texas) showed a high number of institutions failing to pass the clinical acceptable tolerances limits of $7 \%$ dose difference and/or $4 \mathrm{~mm}$ distance to agreement [1]. A deviation of $5 \%$ in delivered dose could affect the tumor control probability by $10 \%-20 \%$ and normal tissue complication by $20 \%-30 \%$ [2]. At the same time, vendors may provide the "golden beam data" for radiation beam commissioning, which may also be different from Linac to Linac [3] [4] [5] [6] [7]. Clinical scenarios could also exist when certain patients may need to be transferred to the other "twin" Linac during one Linac down time. However, a perfect beam matching data between two Linacs may not exist on those machines that are so-called "twined". Different Linacs may also require different dosimetry tolerances. For example, the Linac for stereotactic radiosurgery (SRS) or stereotactic body radiosurgery (SBRT) will need tighter tolerances for annual QA as recommended by the America Association of Physicist in Medicine (AAPM) task group (TG) report 142 [8]. Although, the AAPM has published several TG reports on Linac's annual QA tolerances [9] [10] [11], questions still exist on how much of these uncertainties can affect the quality of the beam profiles and patient dosimetry.

As of today, limited literature exists to provide answers to the above questions. Previous studies are mainly focusing on beam dosimetry comparison between Linacs. Marshall studied the matching of the $6 \mathrm{MV}$ photon beam characteristics for two dissimilar Linacs, one with a new prototype flattening filter and found that the percentage depth values agreed within $1.3 \%$, and beam profiles agreed within $1 \%$, and the wedge transmission factors agreed within $1 \%$. He concluded that patients can be transferred among these matched machines [7]. Watts did comparative measurements for six Varian model 2100C Linacs (Varian Medical Systems, Palo Alto, CA) and found that the beam parameters were similar $(\leq 2 \%)$ and concluded that a standard data set could be used to validate new commissioning data [12]. Hinson et al. compared the energy spectra of four dosimetrically matched Linacs and found the percentage depth dose curves were matched within 1.3\% [13]. Sjostrom et al. compared eight Varian iXLinacs and found that the vendor's acceptance criteria weren't strict enough [14]. Bhangle et al. analyzed two Siemens ONCOR Impression PlusLinacs (Siemens, Berlin, Germany) us- 
ing gamma analysis method and found that the maximum point dose difference was up to $1.5 \%$ [15]. Beyer compared three commissioned TrueBeam Linacs to aTrilogy and Clinac 2100 Linacs (Varian Medical Systems, Palo Alto, CA) and found the accepted clinical tolerance of $\pm 2 \%$ [16]. In summary, the above mentioned studies have quantified the radiation characteristics between different versions/models of Linacs and found dosimetric agreements within $2 \%$, but literature data are scarce on the dosimetric consequences from setup uncertainties.

Although TG 142 has provided strict tolerances for collecting beam data during annual QA and machine commissioning, how much of these uncertainties propagating into patient's treatment plan quality assurance is still unclear. The results from this study provided readers that clarification. It also provided the general information on the dose distribution and its effects for different clinical situations due to patient's set up errors. In this study, we investigated three common potential setup uncertainties in gantry rotation, isocenter and SAD during the Linac annual QA; and evaluated how these setup uncertainties propagate into the quality of the beam profiles using gamma analysis method. By systematically simulating various situations, we intend to evaluate the quantitative dependence of dosimetric characteristics on these setup uncertainties. We further discuss the sensitivity of these beam profile tests to reveal setup uncertainties, which provides data for clinical physicists to diagnose or troubleshoot during annual QA procedures.

\section{Materials and Methods}

\subsection{Clinical Situation Simulated}

A water tank is commonly used to collect radiation beam data therefore any setup uncertainties associate with it can propagate into the quality of the patient's treatment plans. The water tank can be assumed as a rigid body and the transformations caused by the uncertainties during the set up can be calculated using Equation (1)

$$
\mathrm{X}^{\prime}=\mathrm{s}[\mathrm{RX}+\mathrm{T}]
$$

where $\mathrm{X}^{\prime}$ is the transformed object; $\mathrm{X}$ is the original object; $\mathrm{R}$ is the rotation component; $\mathrm{T}$ is the position component and s the scaling factor [17]. For machine QA applications, the deviation from the beam incoming angle will affect the $\mathrm{R}$ component, the offset from the isocenter alignment will affect the $\mathrm{T}$ component and the error from the SSD indicator will affect the scaling factor. Therefore in this study, we simulated three set up scenarios as illustrated in Figure 1(a). Each scenario was created with one uncertainty introduced while the other parameters remained constant. We have pre-selected $\mathrm{R}$ to be $0.25,0.5,1,2,3$ degree; $\mathrm{T}$ to be $1,2,4$, and $6 \mathrm{~mm}$ shifts on the crossline plane; and the s component is the scaling factor for Source-axis distance (SAD) changes by $-0.5,0.3,2$, and $3 \mathrm{~cm}$ from $100 \mathrm{~cm}$.

\subsection{Beam Data Simulated and Collected}

Data was simulated using a $60 \times 60 \times 60 \mathrm{~cm}^{3}$ solid cube phantom (Figure $1(\mathrm{~b})$ ) created 


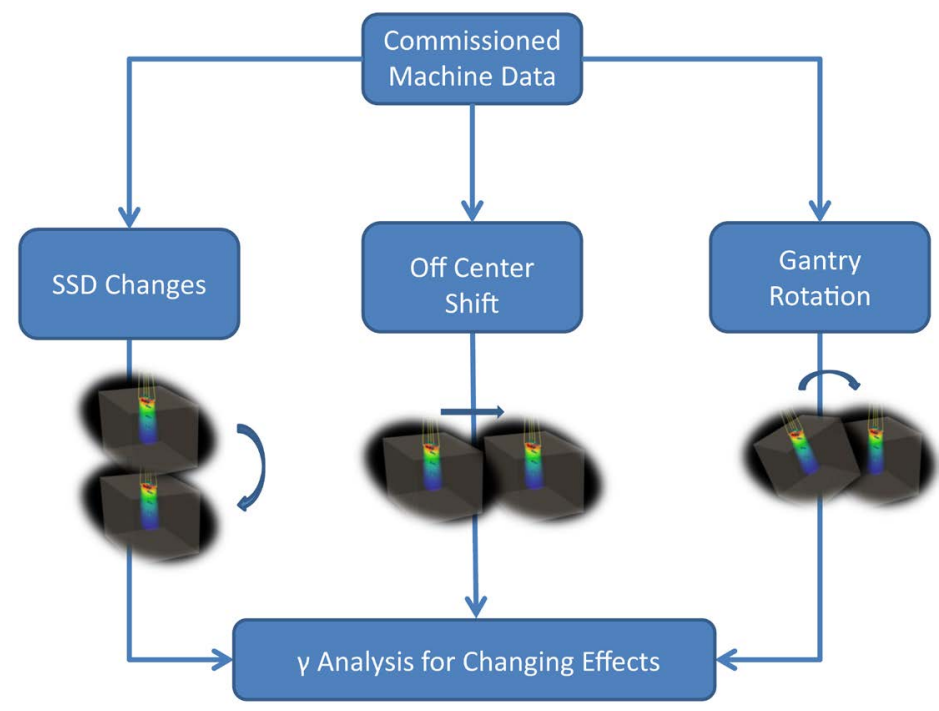

(a)

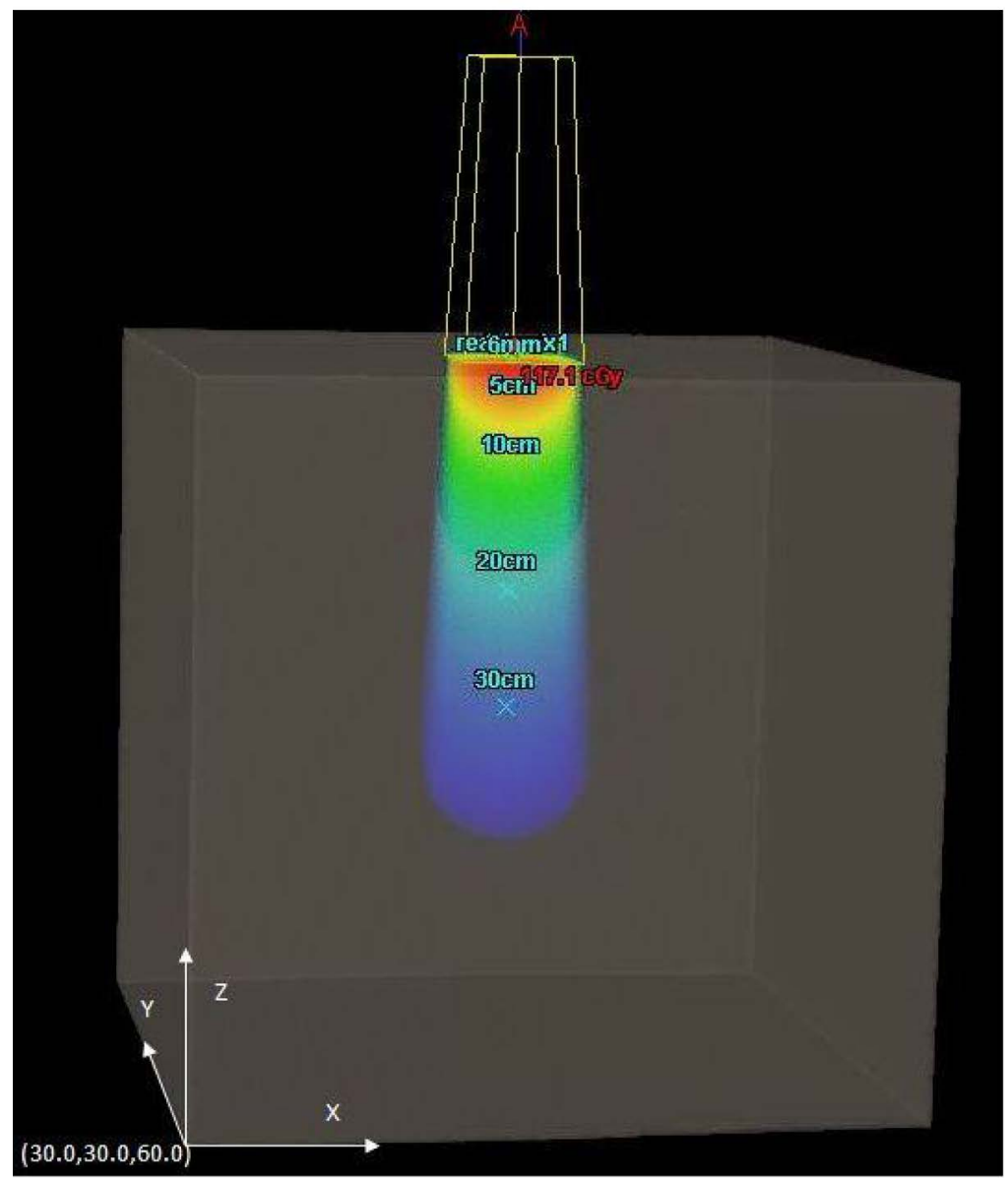

(b)

Figure 1. (a) Three clinical setup scenarios. Each scenario was created with one uncertainty introduced while the other parameters remained constant. A gamma analysis method was applied to study the changing effects (b) A $60 \times 60 \times 60 \mathrm{~cm}^{3}$ solid water cube phantom used for the simulation. 
in Varian Eclipse treatment planning software (TPS) version 11.0 (Varian Medical Systems, Palo Alto, CA) to replicate a 3D water tank. The Hounsfield unit (HU) for the cube was manually assigned to zero. A commissioned beam data set with three energies $6 \mathrm{MV}, 6 \mathrm{MV}$ flattening filter free (FFF), and $10 \mathrm{MV}$-FFF was used.

For each scenario, the beam data which including crossline, diagonal and percent depth dose (PDD) curves were simulated individually with each uncertainty parameter at different field sizes: $2 \times 2 \mathrm{~cm}^{2}, 10 \times 10 \mathrm{~cm}^{2}, 25 \times 25 \mathrm{~cm}^{2}$ and $40 \times 40 \mathrm{~cm}^{2}$ and different depths: $d_{\max }, 5,10,20$ and $30 \mathrm{~cm}$. To create the beam data in Eclipse, a single $3 \mathrm{D}$ static field was applied to the phantom where gantry, collimator and couch were set to zero. The volumetric dose was calculated using the analytical anisotropic algorithm (AAA). A dose line profile function was used and applied at the center of the phantom from the top $(0.0,0.0,0.0 \mathrm{~cm})$ to the bottom $(0.0,0.0,60.0 \mathrm{~cm})$ positions to create a PDD curve. For the crossline and diagonal profiles, the dose line function was applied across from left $(30.0,0.0,0.0 \mathrm{~cm})$ to right $(-30.0,0.0,0.0 \mathrm{~cm})$ and diagonally from position $-30.0,30.0,0.0 \mathrm{~cm}$ to position $30.0,-30.0,0.0 \mathrm{~cm}$ through the phantom's center at different depths. Each profile was calculated using a step size of $0.3 \mathrm{~mm}$ which is the smallest step size allowed in Eclipse TPS. A total of 3 scenarios and each scenario contained from 4 to 5 cases as listed in Table 1. Gantry angles, SAD and beam isocenter positions were manually adjusted under the field property tab for each case.

Table 1. Beam data (crossline, diagonal and PDD) for a total of 3 scenarios and each scenario has from 4 to 5 cases to study. An additional set of data was created without any deviations to be used as baseline.

\begin{tabular}{|c|c|c|c|}
\hline & Gantry Uncertainties & Isocenter Uncertainties & SAD Uncertanties \\
\hline & (degree) & $(\mathrm{mm})$ & $(\mathrm{cm})$ \\
\hline \multicolumn{4}{|c|}{ Scenario 1: Gantry Rotation } \\
\hline Case 1 & 0.25 & 0 & 100 \\
\hline Case 2 & 0.5 & 0 & 100 \\
\hline Case 3 & 1 & 0 & 100 \\
\hline Case 4 & 2 & 0 & 100 \\
\hline Case 5 & 3 & 0 & 100 \\
\hline \multicolumn{4}{|c|}{ Scenario 2: Isocenter off center } \\
\hline Case 1 & 0 & 1 & 100 \\
\hline Case 2 & 0 & 2 & 100 \\
\hline Case 3 & 0 & 4 & 100 \\
\hline Case 4 & 0 & 6 & 100 \\
\hline \multicolumn{4}{|c|}{ Scenario 3: SAD Deviation } \\
\hline Case 1 & 0 & 0 & 99.5 \\
\hline Case 2 & 0 & 0 & 100.3 \\
\hline Case 3 & 0 & 0 & 102 \\
\hline Case 4 & 0 & 0 & 103 \\
\hline Baseline & 0 & 0 & 100 \\
\hline
\end{tabular}


A total of 13 beam data sets (cases 1 - 13) were created for three energies (6 MV, 6 MV-FFF and $10 \mathrm{MV}$-FFF). An additional set was created without any deviations to be used as baseline as shown in Table 1. Table 2 listed an example of the data set created for each scenario. A total of 45 profiles and PDDs were created for each case and energy. A total of 263 profiles were created for all situations.

\subsection{Gamma Analysis}

The beam data for each setup uncertainty was quantitatively compared to baseline using the gamma analysis method established by Low et al. [18]. A gamma analysis program was created using both Microsoft Excel version 2007 (Microsoft Corp., Redmond, WA) and Matlabversion 8.3.0.532 (MathWorks, Natick, MA). Both versions yielded the same results however we found it more difficult to work with Matlab due to issues with data importing and formatting therefore the majority of the analysis were calculated on

Table 2. An example (Scenario 1 and Case 1) of profiles and PDD data for 3 different energies at 4 different field sizes and 5 different depths.

\begin{tabular}{|c|c|c|c|c|c|}
\hline \multicolumn{6}{|c|}{ Scenario 1 , Case 1 : Gantry rotated 0.25 degree, Isocenter at 0 and SAD at 100} \\
\hline \multicolumn{2}{|c|}{$6 \mathrm{MV}$} & \multicolumn{2}{|c|}{$6 \mathrm{MV}-\mathrm{FFF}$} & \multicolumn{2}{|c|}{$10 \mathrm{MV}$-FFF } \\
\hline Field Size $\left(\mathrm{cm}^{2}\right)$ & Depth (cm) & Field Size $\left(\mathrm{cm}^{2}\right)$ & Depth $(\mathrm{cm})$ & Field Size $\left(\mathrm{cm}^{2}\right)$ & Depth $(\mathrm{cm})$ \\
\hline $2 \times 2$ & 1.5 & $2 \times 2$ & 1.5 & $2 \times 2$ & 2.1 \\
\hline $2 \times 2$ & 5 & $2 \times 2$ & 5 & $2 \times 2$ & 5 \\
\hline $2 \times 2$ & 10 & $2 \times 2$ & 10 & $2 \times 2$ & 10 \\
\hline $2 \times 2$ & 20 & $2 \times 2$ & 20 & $2 \times 2$ & 20 \\
\hline $2 \times 2$ & 30 & $2 \times 2$ & 30 & $2 \times 2$ & 30 \\
\hline $10 \times 10$ & 1.5 & $10 \times 10$ & 1.5 & $10 \times 10$ & 2.1 \\
\hline $10 \times 10$ & 5 & $10 \times 10$ & 5 & $10 \times 10$ & 5 \\
\hline $10 \times 10$ & 10 & $10 \times 10$ & 10 & $10 \times 10$ & 10 \\
\hline $10 \times 10$ & 20 & $10 \times 10$ & 20 & $10 \times 10$ & 20 \\
\hline $10 \times 10$ & 30 & $10 \times 10$ & 30 & $10 \times 10$ & 30 \\
\hline $25 \times 25$ & 1.5 & $25 \times 25$ & 1.5 & $25 \times 25$ & 2.1 \\
\hline $25 \times 25$ & 5 & $25 \times 25$ & 5 & $25 \times 25$ & 5 \\
\hline $25 \times 25$ & 10 & $25 \times 25$ & 10 & $25 \times 25$ & 10 \\
\hline $25 \times 25$ & 20 & $25 \times 25$ & 20 & $25 \times 25$ & 20 \\
\hline $25 \times 25$ & 30 & $25 \times 25$ & 30 & $25 \times 25$ & 30 \\
\hline $40 \times 40$ & 1.5 & $40 \times 40$ & 1.5 & $40 \times 40$ & 2.1 \\
\hline $40 \times 40$ & 5 & $40 \times 40$ & 5 & $40 \times 40$ & 5 \\
\hline $40 \times 40$ & 10 & $40 \times 40$ & 10 & $40 \times 40$ & 10 \\
\hline $40 \times 40$ & 20 & $40 \times 40$ & 20 & $40 \times 40$ & 20 \\
\hline $40 \times 40$ & 30 & $40 \times 40$ & 30 & $40 \times 40$ & 30 \\
\hline
\end{tabular}


Excel. The dose difference (DD) and distance to agreement (DTA) distribution were used to evaluate the differences between each data point to baseline. Gamma analysis parameters of $1 \mathrm{~mm} / 1 \%, 2 \mathrm{~mm} / 2 \%$ and $3 \mathrm{~mm} / 3 \%$ were used. The compared points need to have a gamma of less than 1 to pass. The compared profiles need to have a gamma passing rate of $>90 \%$ to pass which means that $90 \%$ of the compared points in the profiles need to be less than 1 .

\section{Results}

\subsection{MV-FFF Beam Data Analysis}

Depicted in Figures 2(a)-2(f) are results of the gamma analysis using $1 \mathrm{~mm} / 1 \%$ criteria for 6 MV-FFF's crossline and diagonal profiles for all three scenarios. Please note that the gamma passing rate is the average of all four field sizes $(2 \times 2,10 \times 10,25 \times 25$, and $40 \times 40 \mathrm{~cm}^{2}$ ). Figures $3(\mathrm{a})-3(\mathrm{c})$ displayed the average dose difference from baseline for $6 \mathrm{MV}$-FFF's PDD at different field sizes.

For gantry rotation scenario, the analysis (Figure 2(a) and Figure 2(b)) showed a decrease in gamma passing rate for both crossline and diagonal profiles as discrepancy increases. An increase in dose difference was also seen on PDD. A $90 \%$ passing rate or better was found for gantry tilted up to 3 degree for all depths in crossline and diagonal profiles. For gamma passing rate of $95 \%$ or greater, a decrease in gamma passing rate for crossline profiles at deeper depths were seen however, it didn't affect the larger diagonal profiles as much. This is reasonable as the change of gantry angle has more drastic effect on the cross profiles than the diagonal ones. Therefore, the gamma analysis of cross profile is a sensitive test for gantry rotation setup uncertainty. In contrast, the dose difference of PDD is not a sensitive test for this scenario. Figure 3(a) shows that the dose difference was less than $1 \%$ for gantry tilted by as large as 2 degree.

For isocenter scenario, a $96 \%$ passing rate or higher were found where isocenter was mis-aligned up to $2 \mathrm{~mm}$ for crossline and $4 \mathrm{~mm}$ for diagonal profiles (Figure 2(c) and Figure 2(d)). For a $4 \mathrm{~mm}$ off center, a gamma passing rate of less than $90 \%$ at the depth between $\mathrm{d}_{\max }$ to $5 \mathrm{~cm}$ was found only on crossline profiles. Dose difference of less than $1 \%$ was found on PDD curves for all isocenter mis-alignment cases (Figure 3(b)). This indicates neither the profile gamma analysis nor PDD dose difference are a sensitive test for this isocenter alignment scenario.

For SAD uncertainty, Figures 2(e)-2(f) showed a gamma passing rate of $98 \%$ or more for all depths for SAD at $100.3 \mathrm{~cm}(0.3 \mathrm{~cm}$ off the baseline of $100.0 \mathrm{~cm})$. For SAD at $99.5 \mathrm{~cm}$, the gamma passing rate dropped down to less than $90 \%$ and to as far as $80 \%$ at depths between $\mathrm{d}_{\max }$ to $13 \mathrm{~cm}$ in both crossline and diagonal profiles. For SAD at $102.0 \mathrm{~cm}$ and $103.0 \mathrm{~cm}$, the gamma passing rate decreased down to $65 \%$ from $\mathrm{d}_{\max }$ to depth of $17 \mathrm{~cm}$ and $24 \mathrm{~cm}$ respectively for crossline and diagonal. Dose differences from $1 \%$ up to $5.3 \%$ were found in PDD for depths from $d_{\max }$ to 15,17 and $25 \mathrm{~cm}$ for $\mathrm{SAD}$ at $99.5,102.0$ and $103.0 \mathrm{~cm}$ respectively. Using a $2 \% / 2 \mathrm{~mm}$ and $3 \% / 3 \mathrm{~mm}$ gamma parameters as shown in Figure 4(a), Figure 4(b), an improvement in gamma passing rate was found in all cases, especially for $99.5 \mathrm{~cm}$ where $\geq 90 \%$ passing rate were found 


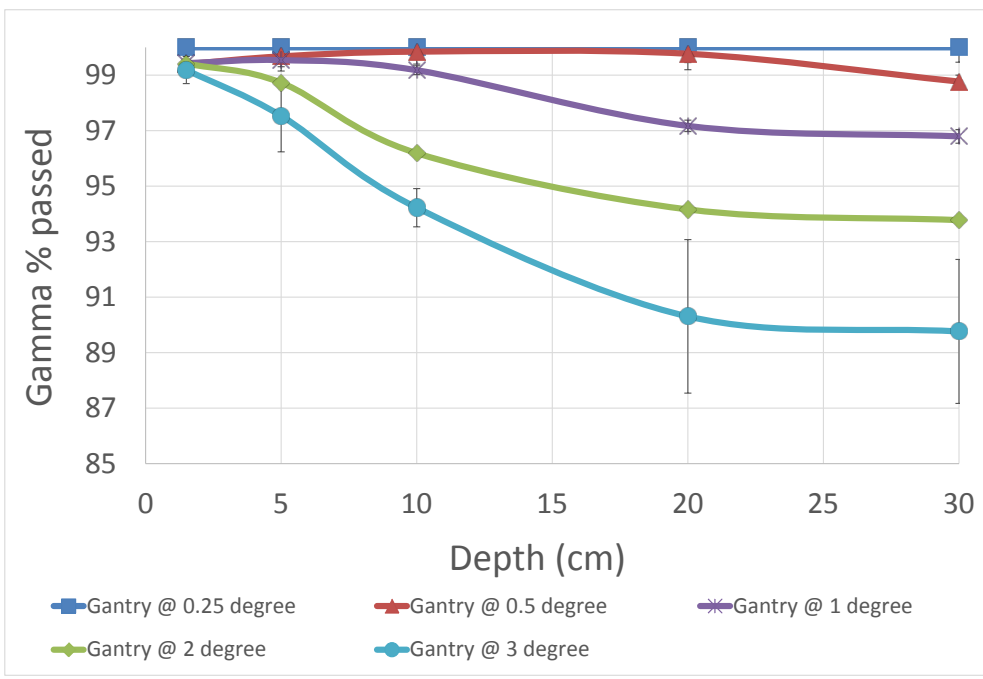

(a)

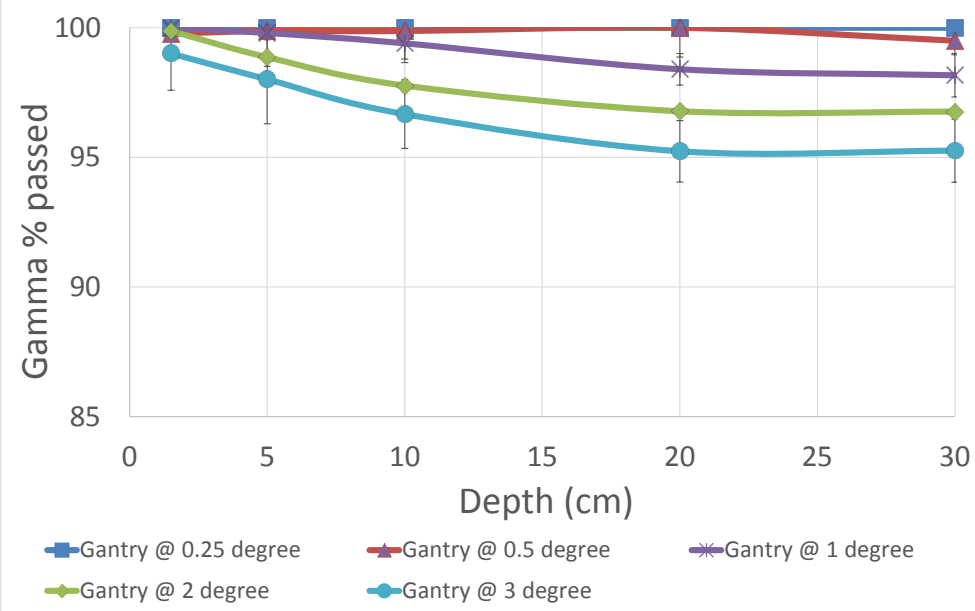

(b)



(c) 




(d)

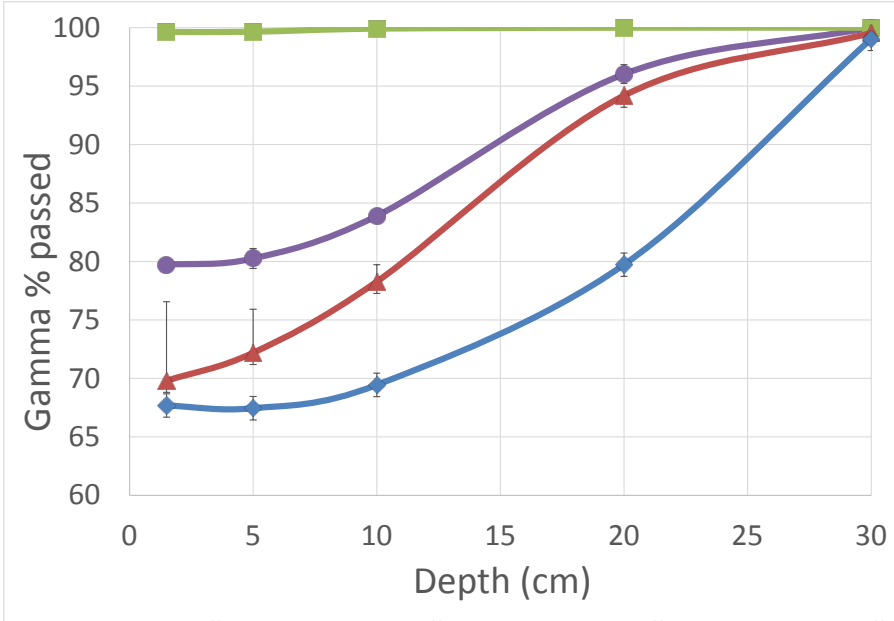

-SAD @ 99.5 cm off =SAD @ 100.3 cm off -SAD @ $102.0 \mathrm{~cm}$ off $\rightarrow$ SAD @ $103.0 \mathrm{~cm}$ off

(e)

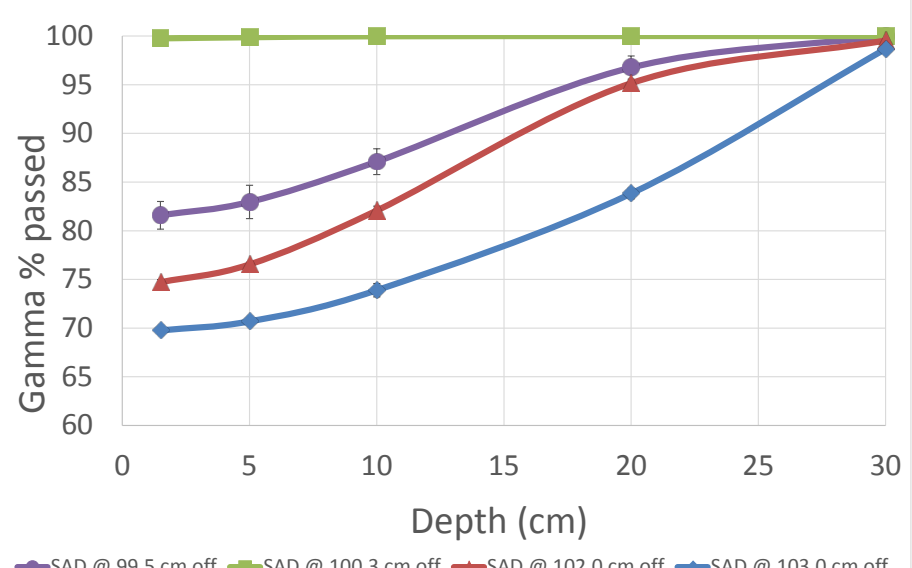

(f)

Figure 2. Gamma analysis using $1 \% / 1 \mathrm{~mm}$ for $6 \mathrm{MV}$-FFF energy (a) Crossline profile for gantry rotation from 0.25 - 3.0 degree; (b) Diagonal profile for gantry rotation from 0.25 - 3.0 degree; (c) Crossline profile for isocenter off center by 1,2, 4 and $6 \mathrm{~mm}$; (d) Diagonal profile for isocenter off center by 1, 2, 4 and $6 \mathrm{~mm}$; (e) Crossline profile for SAD deviation at 99.5, 100.3, 102.0 and $103.0 \mathrm{~cm}$; (f) Diagonal profile for SAD deviation at 99.5, 100.3, 102.0 and $103.0 \mathrm{~cm}$. 


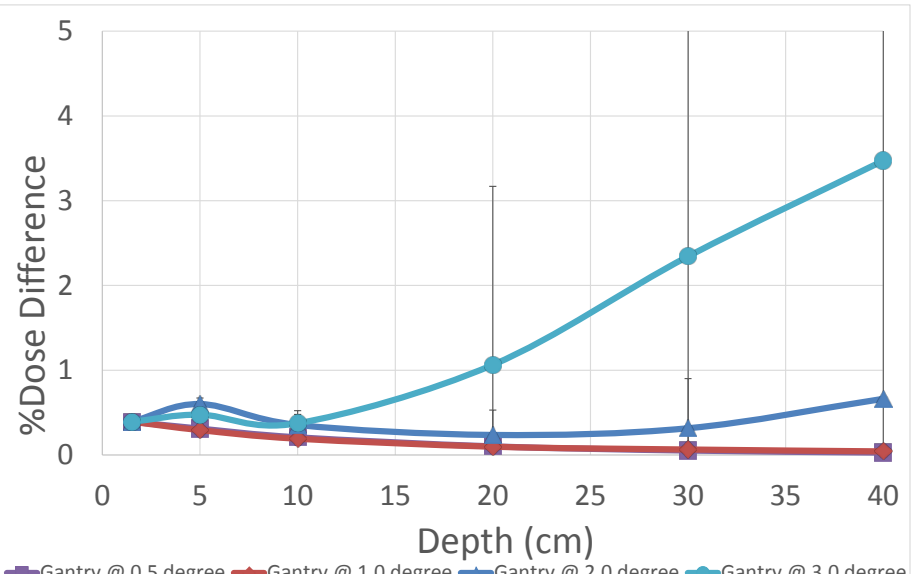

Gantry @ 0.5 degree —Gantry @ 1.0 degree _-Gantry @ 2.0 degree-a-Gantry @ 3.0 degree

(a)

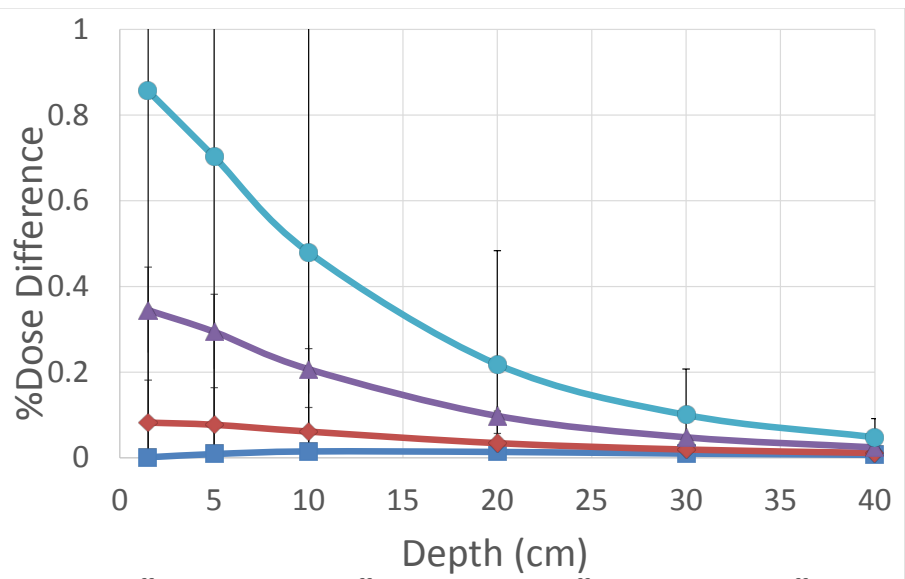

$\leftarrow 1 \mathrm{~mm}$ Off Center $\prec 2 \mathrm{~mm}$ Off Center $-4 \mathrm{~mm}$ Off Center $\prec-6 \mathrm{~mm}$ Off Center

(b)

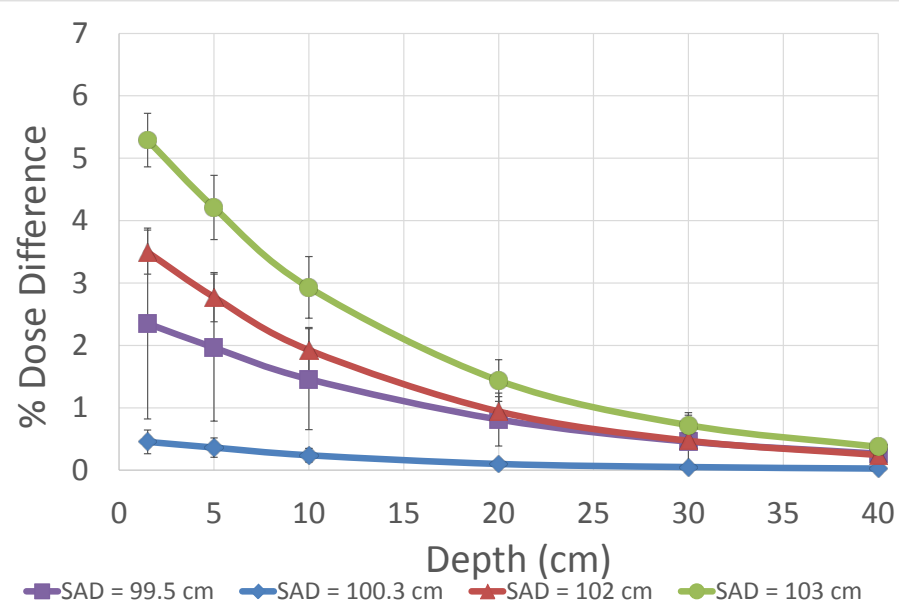

(c)

Figure 3. Percent dose differences for PDD for $6 \mathrm{MV}$-FFF energy (a) gantry rotation from 0.25 - 3.0 degree; (b) isocenter off center by 1, 2, 4 and $6 \mathrm{~mm}$ and (c) SAD deviation at 99.5, 100.3, 102.0 and $103.0 \mathrm{~cm}$. 


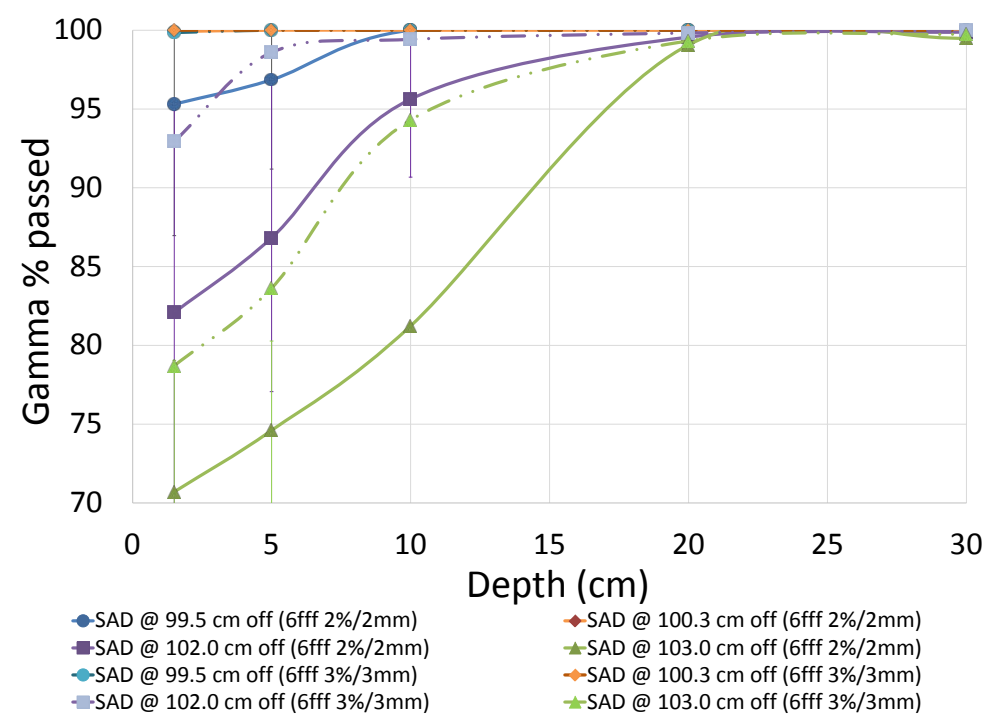

(a)

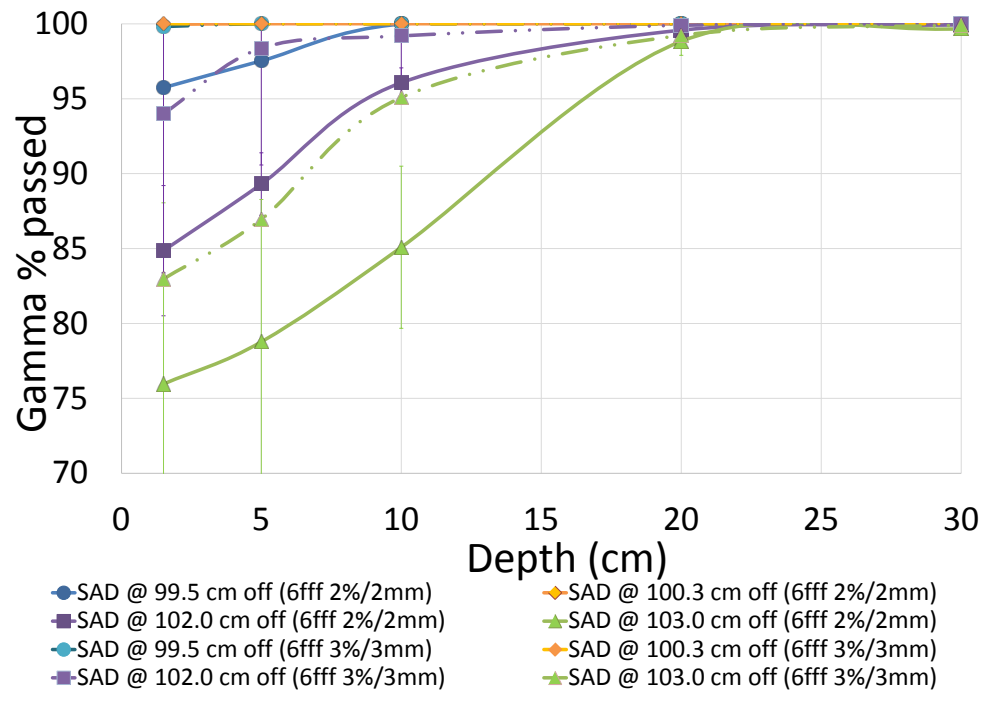

(b)

Figure 4. Gamma analysis using 2\%/2mm (solid) and 3\%/3mm (dashed) for 6 MV-FFF energy (a) Crossline profile for SAD deviation at 99.5, 100.3, 102.0 and $103.0 \mathrm{~cm}$ (b) Diagonal profile for SAD deviation at 99.5, 100.3, 102.0 and $103.0 \mathrm{~cm}$.

for all depths. Profiles with SAD at 102.0 and $103.0 \mathrm{~cm}$ failed mostly at shallow depths from dmax up to $15 \mathrm{~cm}$. The improved passing rates for larger criteria not only have proven our analysis methodology, but also shown that the sensitivity of this test to catch SAD setup uncertainty depends on the gamma analysis criteria.

Figure 5 is an example of direct comparison between the two profiles using gamma analysis. In this example, majority of the failing points are at the penumbra regions as shown in Figure 5(a), Figure 5(b) for gantry and isocenter scenarios. For SAD scenario, the failing points are at the center regions of the beam (Figure 5(c)). 


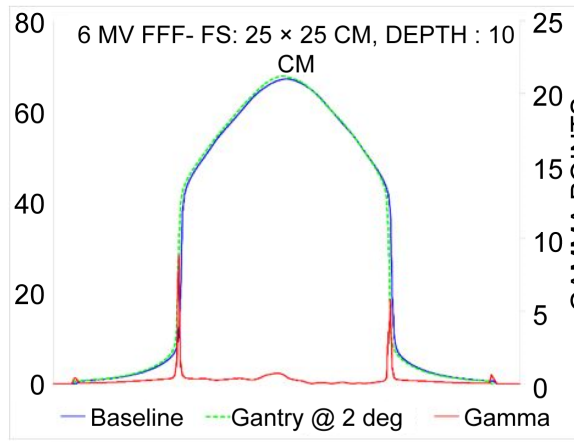

(a)

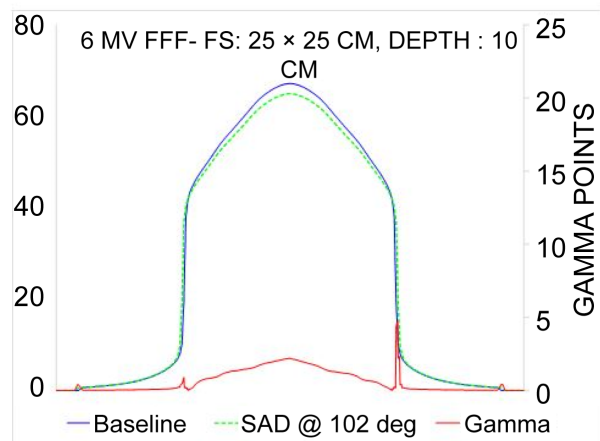

(c)



(b)

Figure 5. Examples of direct comparison between the two profiles (6MV-FFF Field Size: $25 \times 25$ $\mathrm{cm}$, depth at $10 \mathrm{~cm}$ ) using gamma analysis for different scenarios. For gantry and isocenter misalignment uncertainty scenarios, majority of the failing points are at the penumbra regions as shown in Figure 5(a) and Figure 5(b). For SAD uncertainty scenarios, the failing points are at the center of the region of the beam (Figure $5(c)$ ).

\section{2. $6 \mathrm{MV}$ and 10 MV-FFF Beam Data Analysis}

Depicted in Figure 6, Figure 7 are the results of the gamma analysis using $1 \mathrm{~mm} / 1 \%$ with $90 \%$ passing rate criteria for $6 \mathrm{MV}$ and $10 \mathrm{MV}$ FFF energies. Figures $6(\mathrm{a})-6(\mathrm{c})$ are the gamma analysis results of the crossline profiles for all three scenarios. Figures 6(d)-6(f) are the gamma analysis results of the diagonal profiles for all three scenarios. Figures $7(\mathrm{a})-7$ (c) are the percent dose difference calculated for PDD curves for all three scenarios.

For gantry scenario, when $6 \mathrm{MV}$ and $10 \mathrm{MV}$-FFF energies were analyzed, a $\geq 90 \%$ gamma passing rate and $\leq 1 \%$ dose difference were seen on both crossline and diagonal profiles, and PDD curves for gantry tilted up to 2 degree. For 3 degree and greater, the gamma passing rate decreased $\leq 90 \%$ at depths of $\geq 20 \mathrm{~cm}$ for $6 \mathrm{MV}$ and depths of $\geq 12$ $\mathrm{cm}$ for $10 \mathrm{MV}$-FFF beams. For isocenter scenario, a $\leq 90 \%$ gamma passing rate and $\geq 1 \%$ dose difference were seen at depths from $d_{\max }$ to $20 \mathrm{~cm}$ for both energies for all cases. The only case where a decrease in gamma passing rate to less to $75 \%$ and $1.5 \%$ dose difference were seen was on $10 \mathrm{MV}$-FFF beam for $6 \mathrm{~mm}$ off center. For SAD scenario, $\mathrm{SAD}$ at $100.3 \mathrm{~cm}$ was the only case where the gamma passing rate was $\geq 90 \%$ and percent dose difference was less than $1 \%$ for both energies. 


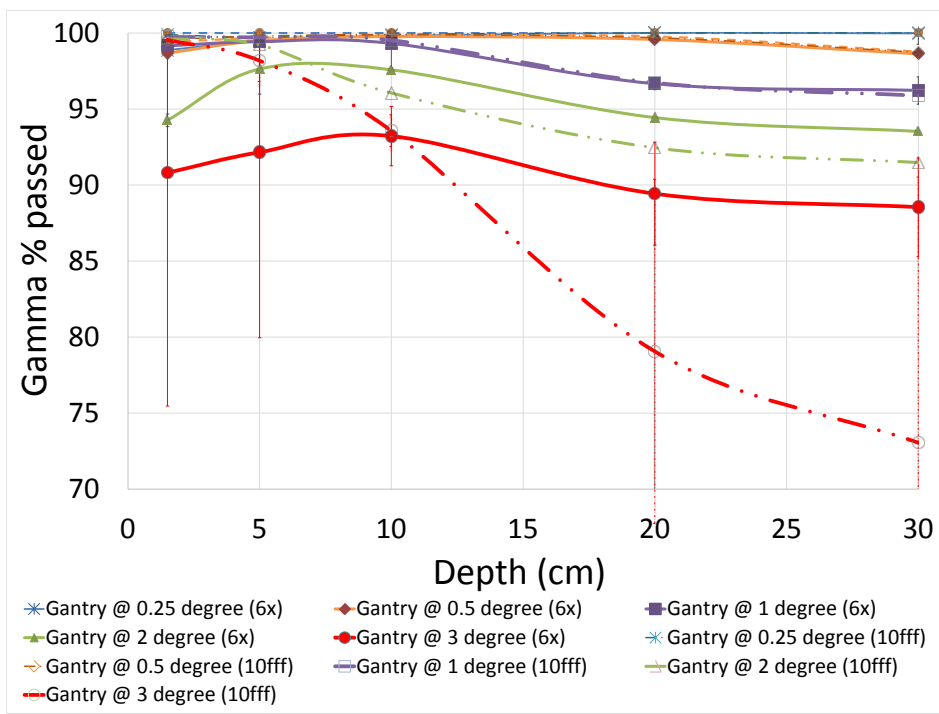

(a)

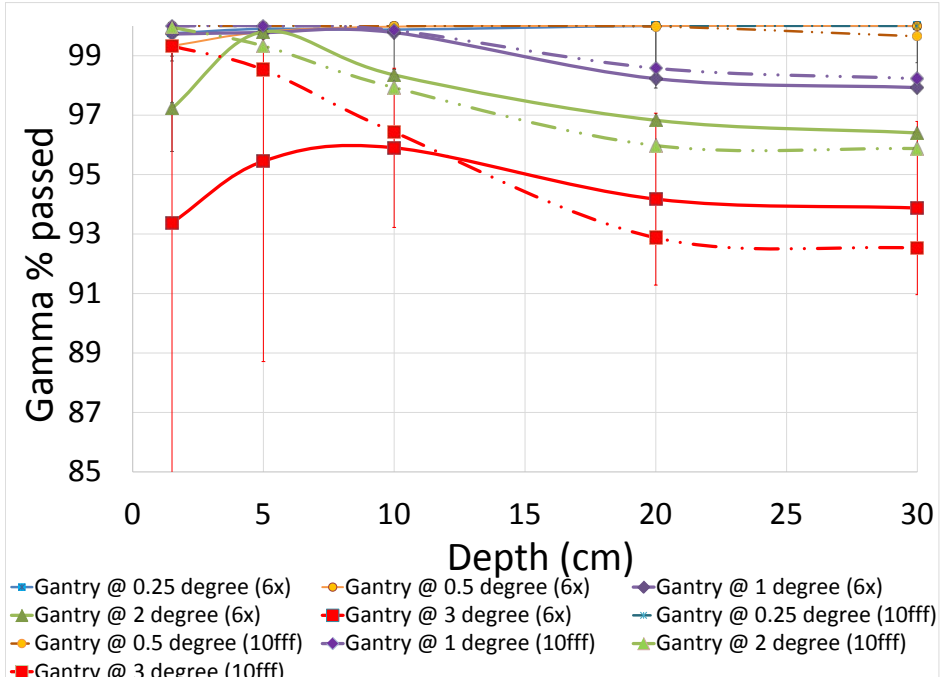

--Gantry @ 3 degree (10fff)

(b)

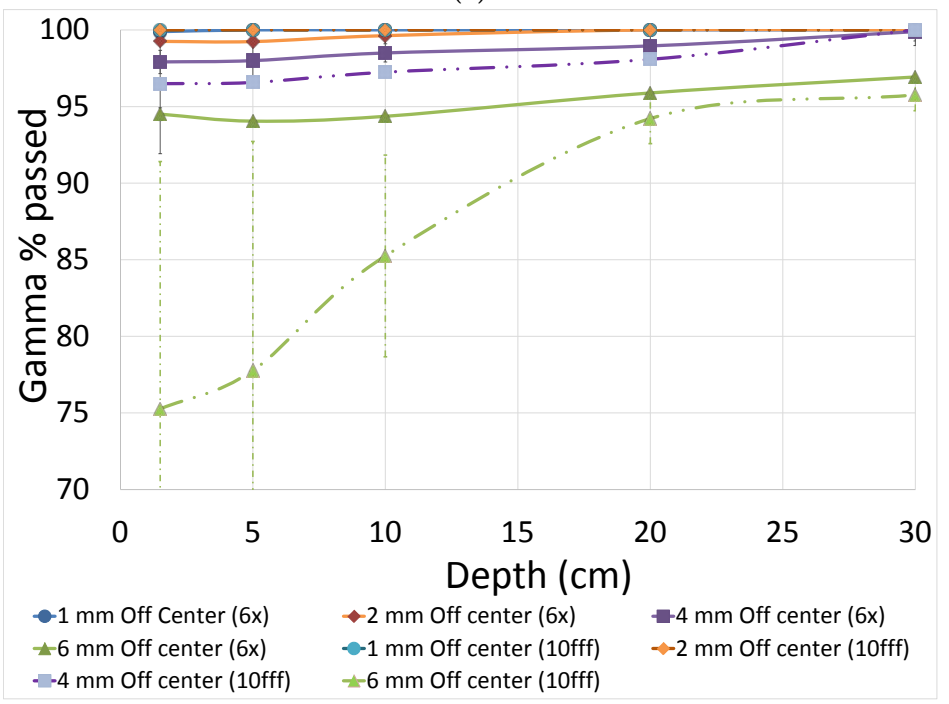

(c) 


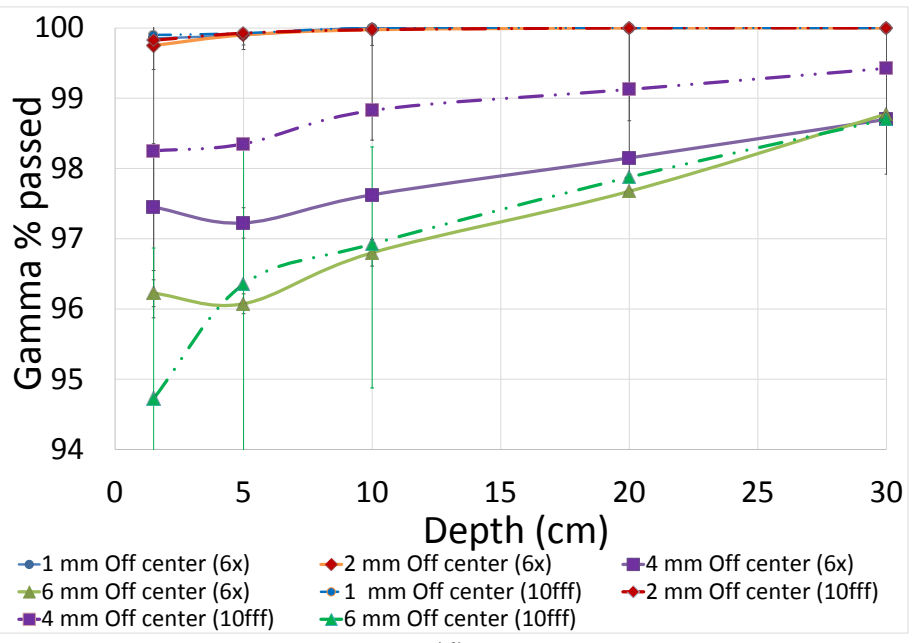

(d)

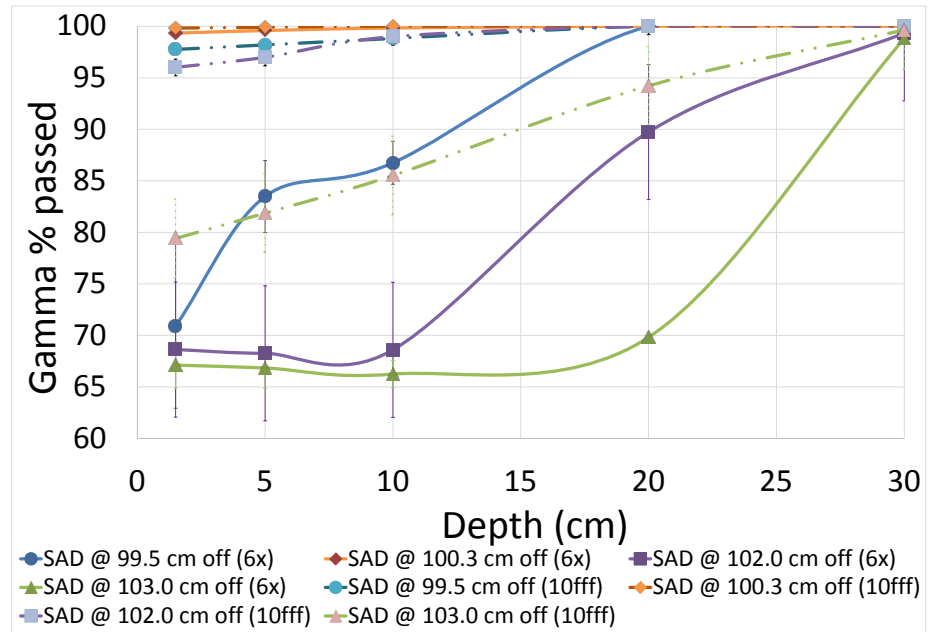

(e)

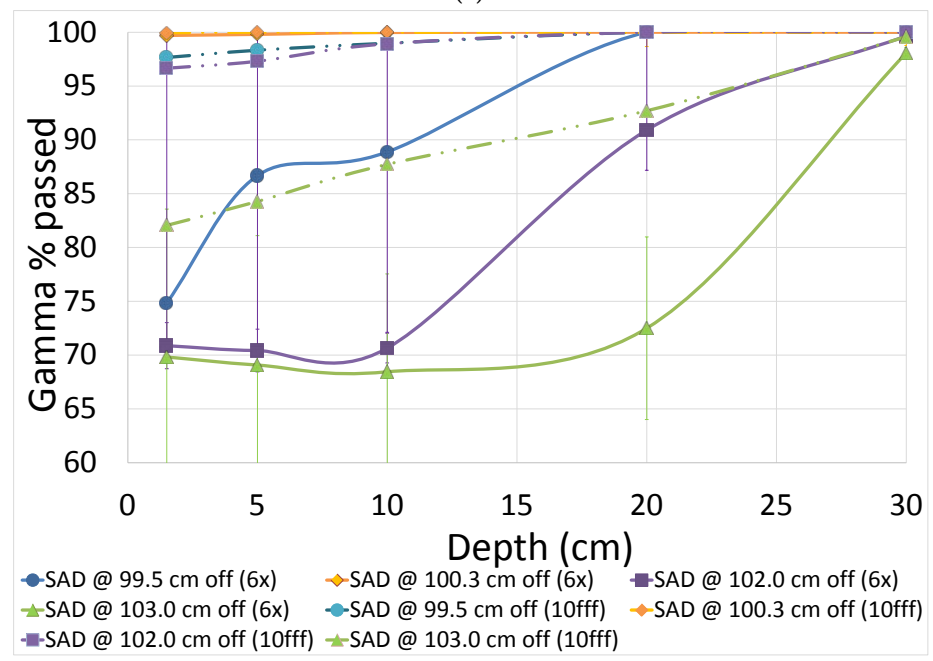

(f)

Figure 6. Gamma analysis using 1\%/1mm for $6 \mathrm{MV}$ (solid) and $10 \mathrm{MV}$-FFF (dashed) energies; (a) Crossline profile for gantry rotation; (b) Diagonal profile for gantry rotation; (c) Crossline profile for isocenter off center; (d) Diagonal profile for isocenter off center; (e) Crossline profile for SAD deviation; (f) Diagonal profile for SAD deviation. 




(a)

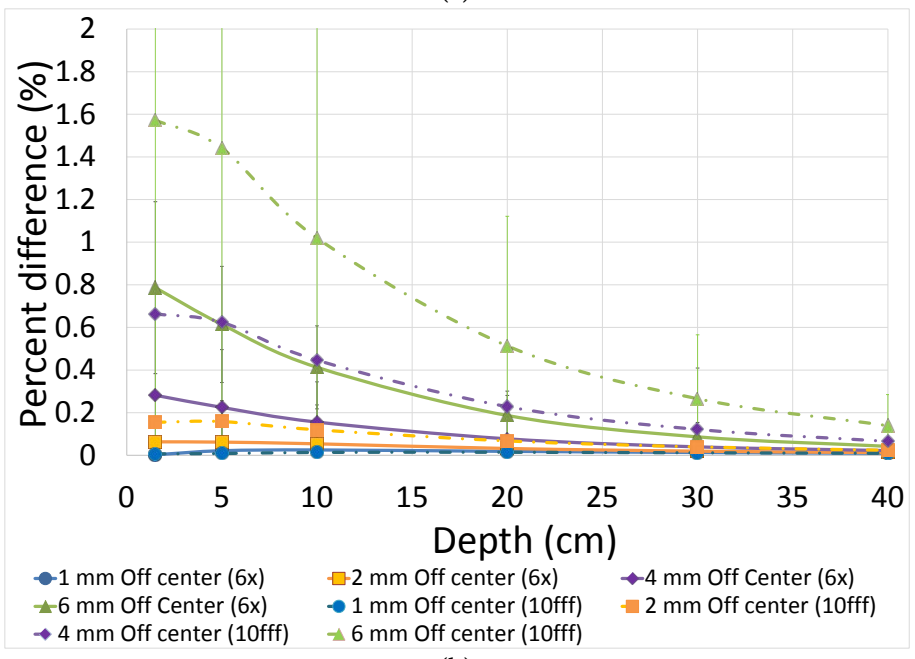

(b)

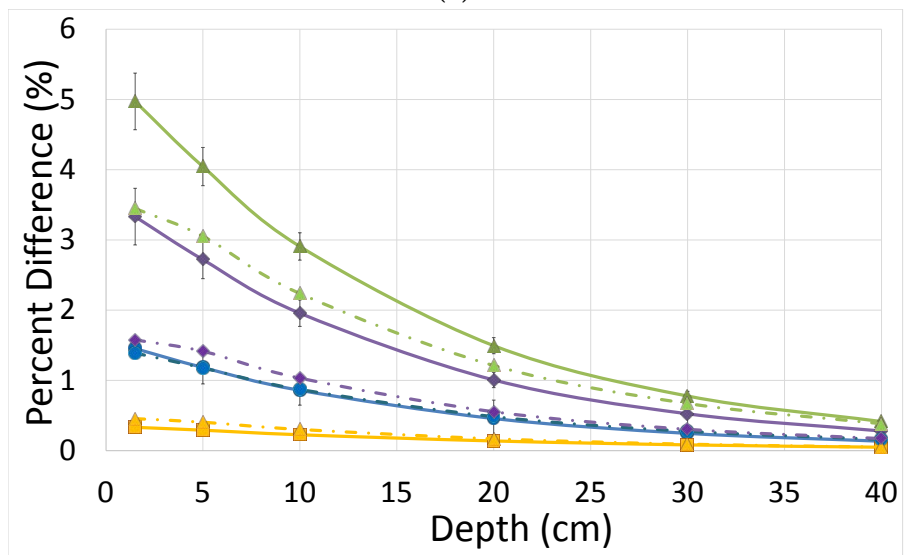

- SAD @ $99.5 \mathrm{~cm}$ off $(6 \mathrm{x}) \quad \square \mathrm{SAD} @ 100.3 \mathrm{~cm}$ off $(6 \mathrm{x}) \rightarrow \mathrm{SAD} @ 102.0 \mathrm{~cm}$ off $(6 \mathrm{x})$ $\rightarrow$ SAD @ $103.0 \mathrm{~cm}$ off $(6 \mathrm{x}) \rightarrow \mathrm{SAD} @ 99.5 \mathrm{~cm}$ off $(10 \mathrm{fff}) \rightarrow \mathrm{SAD} @ 100.3 \mathrm{~cm}$ off $(10 \mathrm{fff})$ $\rightarrow$ SAD @ $102.0 \mathrm{~cm}$ off (10fff) $\rightarrow$ SAD @ $103.0 \mathrm{~cm}$ off (10fff)

(c)

Figure 7. Percent dose differences for PDD for $6 \mathrm{MV}$ (solid) and $10 \mathrm{MV}$-FFF (dashed) energies (a) Gantry rotation from 0.25 - 3.0 degree; (b) Isocenter off center by 1, 2, 4 and $6 \mathrm{~mm}$; (c) SAD deviation by $99.5,100.3,102.0$ and $103.0 \mathrm{~cm}$. 


\section{Discussion}

\subsection{Comparison: Flattening Filter (FF) Beam versus Flattening Filter Free (FFF) Beam}

In general, the gamma analysis is a more sensitive test for unflatten beams as indicated by the larger variations of the passing rate of the $10 \mathrm{MV}$-FFF beams (dashed lines) than those of $6 \mathrm{MV}$ beams (solid lines). When carefully examining each scenario; for gantry rotation, the gamma passing rates are better at shallow depths and decrease as depth increases. The results are as expected since gantry rotations cause larger geometric variations at larger depths. When compared $6 \mathrm{MV}$ to $6 \mathrm{MV}$-FFF beams, the gamma passing rate tended to be better for $6 \mathrm{MV}$-FFF at shallow depths. Please note that direct comparison figures for $6 \mathrm{MV}$ and $6 \mathrm{MV}$-FFF were not shown due to page limitation reasons therefore the $6 \mathrm{MV}$-FFF results can be viewed in Figures 2(a)-2(f), while the 6MV results are in Figures 6(a)-6(f). In general, surface dose at shallow depths are modestly higher for FFF beams compared to FF beams for smaller field sizes. As field size increases, the surface dose increases slower for FFF beams compared to FF beam. As a result, surface dose for FFF beam is comparable or smaller than FF beam for large field sizes [19]. This behavior was also observed during the data analysis of each individual field size. However, the results reported in this paper are the average of the four field sizes and it showed (on average) that surface dose for FFF beam tended to be higher than regular beams as shown in Figure 6(a) and Figure 6(d). For 10 MV-FFF when compared to $6 \mathrm{MV}$, the gamma passing rate is often worsen at deeper depth.

For isocenter scenario, the dosimetric uncertainties were larger at shallower depths. Gamma passing rates were found $\leq 90 \%$ and dose differences were $\geq 1 \%$ for depth from $\mathrm{d}_{\max }$ up to $10 \mathrm{~cm}$ for all energies. $6 \mathrm{MV}$ energy beam has a better gamma passing rate when compared to $6 \mathrm{MV}$-FFF energy beam at shallower depths (6 MV-FFF results presented in Figure 2(c) and Figure 2(d) as compared to $6 \mathrm{MV}$ results presented in Figure 6(b) and Figure 6(e). Up to $6 \mathrm{~mm}$ mis-alignment is allowed for $6 \mathrm{MV}$ beam as compared to $4 \mathrm{~mm}$ for $6 \mathrm{MV}$-FFF beam to achieve a $\geq 90 \%$ gamma pass rate using 1 $\mathrm{mm} / 1 \%$ criteria. The main reason is due to the surface dose is higher for FFF beam than regular flattened beam. When compared $6 \mathrm{MV}$-FFF to $10 \mathrm{MV}$-FFF beams, the gamma and percent dose difference were found to be worsen for $10 \mathrm{MV}$-FFF beams at shallower depths. These results are also expected due to higher energy penetration.

For SAD scenario, SAD's uncertainty up to $3 \mathrm{~mm}$ is allowed for all three energies. The dosimetric uncertainties were also found to be larger at shallower depths. A similar gamma passing trend and percent dose difference were found for $6 \mathrm{MV}$ and $6 \mathrm{MV}$-FFF beams (6 MV-FFF results presented in Figure 2(e) and Figure 2(f) as compared to 6 MV results presented in Figure 6(c) and Figure 6(f)). For 10 MV-FFF beam, the gamma passing rate was found to be better when compared to $6 \mathrm{MV}$-FFF energy at shallow depths. A gamma passing rate $\geq 90 \%$ was found for an uncertainty up to $2 \mathrm{~cm}$ $(\mathrm{SAD}=102 \mathrm{~cm})$ on both crossline and diagonal profiles. However for percent dose difference, dose differences $\leq 1 \%$ were found only on uncertainty of $0.3 \mathrm{~cm}$. 


\subsection{Dosimetric Effects on Treatment Plans}

The main goal of the paper is to provide the readers the quantitative dosimetric effects if a bad beam data set is utilized in the TPS. The author understands that the best way to obtain this information is to create a bad beam data set in the TPS and use it for dose calculation. However, the current version of Eclipse (v.11) has a very strict restriction on the quality of the inputting beam data therefore it prevents us from creating a fake machine using a bad beam data set. Due to this limitation, we have adopted an alternative approach in this study. However, it is noted that the results presented here are not only show the effects due to the geometric deviations from each uncertainty scenario on the quality of the beam data but they also can be applied to study the dose distribution impacts for different clinical situations. For example, the 3D phantom can be viewed as a CT scan of a patient and a static AP beam is applied to treat this particular patient. The accuracy of this treatment is dependent on how accurate the patient is set up and/or how much uncertainty is inherent in the gantry or SSD indicators. If the patient is incorrectly set up by either mis-alignment of the isocenter, SAD or gantry setting which could affect the dose distribution in a similar way as evaluated in this study. Although such AP beam seems simple, it can provide a guideline for more complex treatments of arc and intensity modulated beams. In general, the results could be used as a tool for clinical physicists to evaluate the dose distribution and its effects for different clinical situations due to patient's set up errors. It is noted that due to the limitation of the data used, the study results are not applied to small field dosimetry $(<2 \times 2$ $\mathrm{cm}^{2}$ or SRS beam data).

\section{Conclusion}

We quantified the dependence of dosimetric characteristics on three common setup uncertainties during annual QA procedures. The sensitivities of the gamma analysis and dose difference tests are dependent on the energy and setup uncertainty scenario, and therefore need to be chosen carefully. Gamma analysis of beam profile is a very sensitive test for SAD deviation scenario and can reveal issues for submillimeter setup uncertainty, but it is not as sensitive for the isocenter shift scenario. Even a $4 \mathrm{~mm}$ shift case can still pass with $95 \%$ using $1 \mathrm{~mm} / 1 \%$ gamma analysis criteria. This test is also more sensitive for FFF beams than flat beams.

\section{References}

[1] Ibbott, G., Followill, D., Molineu, A., Lowenstein, J., Alvarez, A. and Roll, J. (2008) Challenges in Credentialing Institutions and Participants in Advanced Technology Multi-Institutional Clinical Trials. International Journal of Radiation Oncology ${ }^{*}$ Biology ${ }^{*}$ Physics, 71, S71-S75. https:/doi.org/10.1016/j.ijrobp.2007.08.083

[2] Chetty, J., Curran, B., Cygler, E., De Marco, J., Ezzell, G. and Faddegon, A. (2007) Report of the AAPM Task Group 105: Issues Associated with Clinical Implementation of Monte Carlo-Based Photon and Electron External Beam Treatment Planning. Medical Physics, 34, 4818-4853. https:/doi.org/10.1118/1.2795842

[3] Kalach, I. and Rogers, O. (2003) Which Accelerator Photon Beams Are Clinic-Like for Ref- 
erence Dosimetry Purposes? Medical Physics, 30, 1546-1555.

https:/doi.org/10.1118/1.1573205

[4] Taylor, C., Tello, M., Schroy, C., Vossler, M. and Hanson, W. (1998) A Generic off Axis Energy Correction for Linac Photon beam Dosimetry. Medical Physics, 25, 662-667. https:/doi.org/10.1118/1.598249

[5] Keall, P., Siebers, V., Jeraj, R. and Mohan, R. (2000) The Effect of Dose Calculation Uncertainty on the Evaluation of Radiotherapy Plans. Medical Physics, 27, 478-484. https:/doi.org/10.1118/1.598916

[6] Ding, G., Duggan, D. and Coffey, C. (2006) Commissioning Stereotactic Radiosurgery Beams Using Both Experimental and Theoretical methods. Physics in Medicine and Biolo$g y$, 51, 2549-2566. https:/doi.org/10.1088/0031-9155/51/10/013

[7] Marshall, M. (1993) Matching the 6-MV Photon Beam Characteristics of Two Dissimilar Linear Accelerators. Medical Physics, 20, 1743-1746. https:/doi.org/10.1118/1.596961

[8] Klein, E., Hanley, J., Bayouth, J., et al. (2009) American Association of Physicists in Medicine Radiation Therapy Committee Task Group 142: Quality Assurance of Medical Accelerators. Medical Physics, 36, 4197-4212. https:/doi.org/10.1118/1.3190392

[9] Svensson, G., Baily, N., Loevinger, R., et al. (1984) American Association of Physicists in Medicine Radiation Therapy Committee Task Group 13: Physical Aspects of Quality Assurance in Radiation Therapy. American Institute of Physics, 6.

[10] Kutcher, J., Coia, L., Gillin, M., et al. (1994) American Association of Physicists in Medicine Radiation Therapy Committee Task Group 40: Comprehensive QA for Radiation Oncology. Medical Physics, 21, 581-618. https:/doi.org/10.1118/1.597316

[11] Das, I., Cheng, W., Watts, R., et al. (2008) American Association of Physicists in Medicine Radiation Therapy Committee Task Group 106: Accelerator Beam Data Commissioning Equipment and Procedures. Medical Physics, 35, 4186-4215. https:/doi.org/10.1118/1.2969070

[12] Watts, R. (1999) Comparative Measurements on a Series of Accelerators by the Same Vendor. Medical Physics, 26, 2581-2585. https:/doi.org/10.1118/1.598796

[13] Hinson, W., Kearns, W., deGuzman, A. and Bourland, D. (2008) Photon Spectral Characteristics of Dissimilar 6 MV Linear Accelerators. Medical Physics, 35, 1698-1702. https:/doi.org/10.1118/1.2900001

[14] Sjostrom, D., Bjelkengren, U., Ottosson, W. and Behrens, C. (2009) A Beam-Matching Concept for Medical Linear Accelerators. Acta Oncologica, 48, 192-200. https:/doi.org/10.1080/02841860802258794

[15] Bhangle, J., Narayanan, S., Kumar, N. and Vaitheeswaran, R. (2011) Dosimetric Analysis of Beam-Matching Procedure of Two Similar Linear Accelerators. Journal of Medical Physics, 36, 176-180. https:/doi.org/10.4103/0971-6203.83497

[16] Beyers, G. (2013) Commissioning Measurements for Photon Beam Data on Three True Beam Linear Accelerators, and Comparison with Trilogy and Clinac 2100 Linear Accelerators. Journal of Applied Clinical Medical Physics, 14, 4077.

[17] Fu, C., Gonzalez, C. and Lee, G. (1987) Control, Sensing, Vision, and Intelligence. McGraw-Hill, New York.

[18] Low, D., Harms, W., Mutic, S. and Purdy, J.A. (1998) A Technique for the Quantitative Evaluation of Dose Distributions. Medical Physics, 25, 656-661. https:/doi.org/10.1118/1.598248

[19] Xiao, Y., Kry, S., et al. (2015) Flattening Filter-Free Accelerators: A Report from the AAPM 
Therapy Emerging Technology Assessment Work Group. Journal of Applied Clinical Medical Physics, 16, 12-29.

Submit or recommend next manuscript to SCIRP and we will provide best service for you:

Accepting pre-submission inquiries through Email, Facebook, LinkedIn, Twitter, etc. A wide selection of journals (inclusive of 9 subjects, more than 200 journals)

Providing 24-hour high-quality service

User-friendly online submission system

Fair and swift peer-review system

Efficient typesetting and proofreading procedure

Display of the result of downloads and visits, as well as the number of cited articles

Maximum dissemination of your research work

Submit your manuscript at: http://papersubmission.scirp.org/

Or contact ijmpcero@scirp.org 